\title{
Influence of implant location on the clinical outcomes of implant abutments: a systematic review and meta-analysis
}

This article was published in the following Dove Press journal: Clinical, Cosmetic and Investigational Dentistry

\author{
Amr G ElHoussiney,' He \\ Zhang, ${ }^{2}$ Jinlin Song, ${ }^{2}$ Ping Ji, ${ }^{2}$ \\ Lu Wang,' Sheng Yang,' \\ 'Department of Prosthodontics, \\ ${ }^{2}$ Chongqing Key Laboratory of Oral \\ Diseases and Biomedical Sciences, \\ Chongqing Municipal Key Laboratory \\ of Oral Biomedical Engineering \\ of Higher Education, College of \\ Stomatology, Chongqing Medical \\ University, Chongqing, China
}

\section{Video abstract}

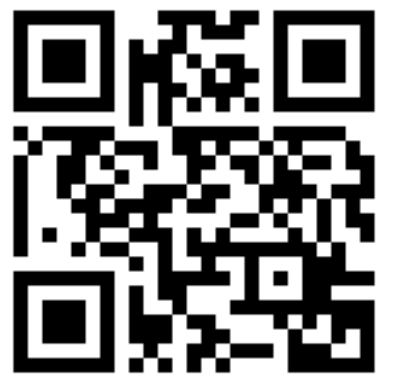

Point your SmartPhone at the code above. If you have a QR code reader the video abstract will appear. Or use: http://youtu.be/01skpVUWtMg
Correspondence: Sheng Yang Department of Prosthodontics, College of Stomatolgy, Chongqing Medical University, 426 North Songshi Road, Yubei, Chongqing, Sichuan 40I I47, China Tel +862388860085 Fax +86 2388860222 Email ysdentist@hospital.cqmu.edu.cn
Purpose: To compare the failure events and incidence of complications of different abutment materials in anterior and posterior regions. Failure was defined as complete loss of the abutment requiring replacement by a new abutment.

Materials and methods: Electronic searches using PubMed/Medline and Google Scholar complemented with manual searches were performed with specific search terms. Searches were restricted to publications in English between January 2006 and March 2016.

Results: A total of 863 and 1,264 implants were inserted in the anterior and posterior regions, respectively, in a total of 1,529 patients. No titanium abutments failed in anterior or posterior regions. On the other hand, $1.6 \%$ of zirconia abutments failed in the anterior region and $1.5 \%$ failed in the posterior region. Technical complications occurred mostly in the posterior region and mostly involved zirconia abutment. Meta-analysis was possible only for zirconia-abutment failure, due to considerable heterogeneity of studies and outcome variables. No significant difference in failure rate was found between anterior and posterior zirconia abutments (risk ratio $1.53,95 \%$ CI $0.49-4.77 ; P=0.47$ ).

Conclusion: This systematic review and meta-analysis showed similar outcomes of different abutment materials when used in anterior and posterior regions in terms of failure events and biological and aesthetic complications. The only significant finding was the increased incidence of technical complications in the posterior region, mostly involving zirconia abutments. Abutment-screw loosening was the most common technical complication.

Keywords: implant abutment, zirconia, titanium, implant location, systematic review

\section{Introduction}

Implant-supported restorations are considered a viable and predictable treatment option for replacing a missing tooth. ${ }^{1,2}$ Important parameters controlling the success of dental implants include proper case selection, surgical technique, and choice of implant-abutment material and design. ${ }^{3}$

Continuous research has led to the evolution of a variety of implant-abutment materials suitable for different clinical situations, in order to achieve ultimate mechanical, biological, and aesthetic outcomes. Implant-abutment materials that are currently used can be divided into two main categories: metal abutments and ceramic abutments. Metal-implant abutments include UCLA abutments, cast metal abutments, and titanium abutments, while ceramic abutments include alumina abutments and zirconia abutments. The clinical outcomes of different implant abutments are influenced by several factors; among these are the method of manufacture, implant-abutment connection, and implant location. ${ }^{4}$ 
The location of the implant in the jaw has a significant influence on the magnitude of load generated by masticatory activity, with the highest biting force occurring at the first molar and the lowest biting force occurring at the incisors. The average values of occlusal forces reported in the anterior region range from $60 \mathrm{~N}$ to $270 \mathrm{~N},{ }^{5}$ while in the premolar and molar regions the mean maximum masticatory forces are 179-294 N, ${ }^{6}$ with much greater loads expected on restorations in patients with functional disorder, such as clenching or bruxism, with forces of 216-890 N.?

The implant location affects the magnitude of masticatory force that the implant abutment is subjected to, affecting the success rate and complications of the implant abutment. Therefore, the clinician's choice of implant-abutment material is influenced by the implant location in the jaw.

Several systematic reviews have reported on the success rate and incidence of technical, biological, and aesthetic complications of implant abutments without any comparison between anterior and posterior regions where all the abutments were pooled together. ${ }^{8,9}$ Other systematic reviews have evaluated the outcomes of implant-abutment material in only the anterior region ${ }^{10}$ or the posterior region. ${ }^{11}$ Therefore, at present there are still limited data reflecting the differences between implantabutment outcomes in anterior and posterior regions. As such, the aim of this study was to investigate the effect of implant location (anterior and posterior) on failure rates and technical, biological, and aesthetic complications of different implant-abutment materials (metal and ceramic). Failure was defined as complete loss of the abutment, requiring replacement by a new abutment.

\section{Materials and methods Focus question}

The PICO (population, intervention, comparison, and outcome) question was stated thus: Does the location of implant restorations (anterior or posterior) have an effect on different implant-abutment materials in terms of survival, mechanical performance, and biological clinical outcomes?

- population: patients treated with dental implant restorations

- intervention: different implant-abutment material

- comparison: anterior and posterior locations

- outcome: survival, mechanical, biological, and aesthetic clinical outcomes.

\section{Literature search}

A Medline (PubMed) and Google Scholar search was performed for clinical studies published in dental journals from January 2006 up to and including March 2016. The search was limited to English-language publications. The electronic search was complemented by manual searching of the bibliographies of the most recent systematic reviews and of all full articles selected to maximize the likelihood of capturing all relevant publications. Key terms included in the search were implant abutment material, zirconia abutments, titanium abutments, gold abutments, UCLA abutments, CadCam abutments, customized abutments, pre-fabricated abutments, alumina abutments, ceramic abutments, and aesthetic abutments.

\section{Study selection}

All publications found were entered into reference-manager software (EndNote, Thomson Reuters Research Soft) to sort selected studies and to discard duplicate references. The criteria for study inclusion were articles in English, articles published in the last 10 years (2006-2016), human clinical studies, at least five patients included in each study, and mean follow-up of at least 1 year. The criteria for study exclusion were articles not pertaining to the inclusion criteria, articles from which data on selected outcome variables could not be directly extracted or calculated, articles pertaining only to one-piece implants, articles with provisional or interim abutments only, animal studies, in vitro experiments, technique, review, or discussion articles, and human clinical studies with fewer than five patients.

All obtained titles identified from the broad electronic and manual search were screened by two independent reviewers to eliminate articles that clearly failed to meet the inclusion and exclusion criteria. Disagreements were resolved by discussion. This was followed by obtaining and screening abstracts of all titles agreed upon by both investigators. Based on the selection of abstracts, articles were obtained in full text. If title and abstract did not provide sufficient information regarding the inclusion criteria, the full report was obtained as well. Again, any disagreement was resolved by discussion. Finally, the selection of full-text articles based on inclusion/ exclusion criteria was made. For this purpose, the Materials and methods, Results, and Discussion sections of these studies were screened. Any questions that came up during the screening were discussed between the two reviewers to aim for consensus.

\section{Quality assessment}

The quality of eligible studies was assessed independently by two authors. Randomized controlled trials (RCTs) were assessed for bias according to the Cochrane Collaboration 
tool. This tool uses six domains (random-sequence generation, allocation concealment, blinding of participants and personnel, blinding of outcome assessment, incomplete outcome data, and selective reporting) to investigate selection, performance, detection, attrition, and reporting biases. ${ }^{12}$

The quality of nonrandomized clinical studies was assessed using the Newcastle-Ottawa scale. This scale includes nine domains using a star system based on three aspects: selection of the study groups (up to 4 points), comparability of the groups (up to 2 points), and exposure or outcome (up to 3 points). ${ }^{13}$ Due to the variability in the quality of the observational studies found in our initial literature search, we considered studies that met five or more of the Newcastle-Ottawa scale score criteria as good quality and included them in our study.

For other types of studies, the quality-assessment tool used was based on an earlier tool developed by den Hartog et al focusing on the criteria: Are the characteristics of the study group clearly described?; Is there a high risk of selection bias?; Are the inclusion and exclusion criteria clearly described?; Is the intervention clearly described?; Are all patients treated according to the same intervention?; Are the outcomes clearly described?; Are adequate methods used to assess the outcome?; Is blinding used to assess the outcome?; Is there a sufficient follow-up?; Can selective loss to follow-up sufficiently be excluded?; Are the most important confounders or prognostic factors identified, and are these taken into consideration with respect to the study design and analysis? ${ }^{14}$ It was decided that studies scoring five or more pluses were considered acceptable.

\section{Data extraction}

A data-extraction sheet was used by two reviewers to extract the relevant data from the included papers. Information on several parameters was recorded: author(s), study design, year of publication, mean follow-up time, abutment manufacturer, number of patients, number of abutments, implant location (anterior or posterior), abutment material, abutment-failure events, implant failures, and incidence of biologic, technical, and aesthetic complications of abutments. Disagreement regarding data extraction was resolved by consensus. Based on the included studies, the number of events of complications or failures was calculated. Where the publication did not provide sufficient information, the corresponding authors were contacted via email.

The anterior region was defined as the area from canine to canine, and the posterior region was defined as the area distal to the canines. Failure was defined as complete loss of the abutment requiring replacement by a new abutment. Technical complications included abutment-screw fracture and abutment-screw loosening. Prosthetic complications included misfit at the implant-abutment junction (gap), fracture of the implant prosthesis, chipping of the veneering ceramic, and loosening of the implant prosthesis. Biological complications included recession, peri-implant bone loss, peri-implant pockets $>3 \mathrm{~mm}$, peri-implant mucosal defects, fistulas, and suppuration on probing.

\section{Statistical analysis}

Meta-analysis was performed using the statistical software package RevMan (version 5.3; Nordic Cochrane Centre, Copenhagen, Denmark) to collect the data, calculate the overall estimated effects, and produce the forest plots. RRs for abutment failure with $95 \%$ CIs were calculated. The pooled effect was considered significant if $P<0.05$. Discrepancies between studies in estimating the effect of treatment were assessed using Cochran's $Q$ test for heterogeneity and associated significant heterogeneity was indicated by $P<0.1$. The $I^{2}$ statistic was used to describe variations across studies due to heterogeneity, and with $I^{2}$ values more than $50 \%$, considerable heterogeneity among studies could be present.

\section{Results Study selection}

The initial database search yielded 1,902 studies after duplicate references had been discarded (Figure 1): 222 potentially relevant titles were selected by two reviewers for abstract evaluation, of which 37 studies were considered for full-text analysis; 14 studies were added after manual searching of the bibliographies of the most recent systematic reviews and of all full articles selected, resulting in a total of 51 articles; 32 studies were finally selected for systematic review and qualitative analysis after screening on the basis of the inclusion and exclusion criteria. The excluded studies and reasons for their exclusion are listed in Table 1. Of the 32 studies, five were eligible for inclusion into a meta-analysis. The 32 studies that met the inclusion criteria are presented in Table 2. A total of seven studies were RCTs, 21 were prospective studies, and the remaining four were retrospective studies.

\section{Quality assessment}

Summaries of the methodological-quality assessment of included studies using the Cochrane risk-of-bias tool and Newcastle-Ottawa Scale are provided in Tables 3 and 4, respectively. For the RCTs, information indicating a low risk of bias was found in two studies. One study revealed unclear 


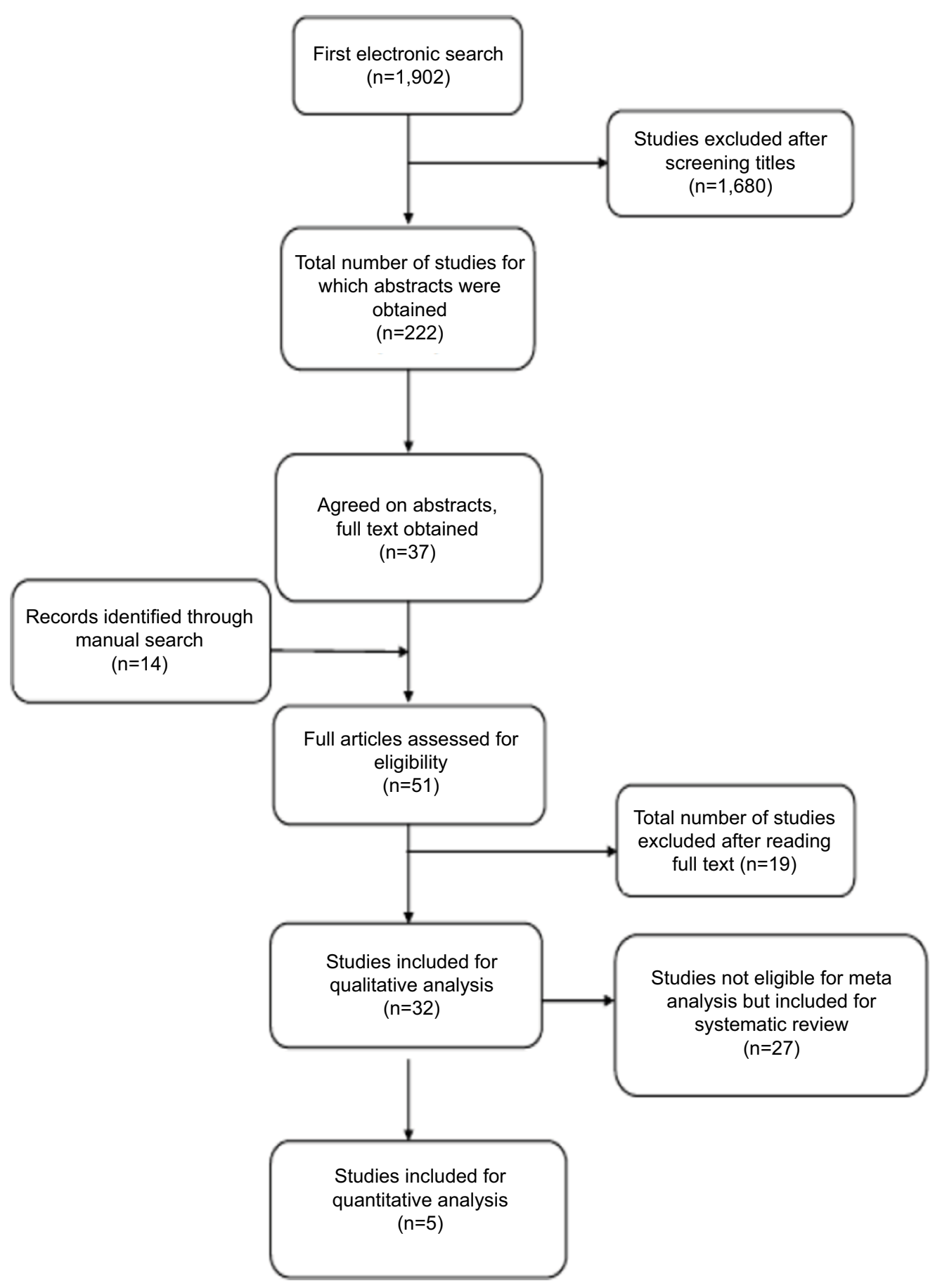

Figure I Search strategy flowchart.

risk of bias in one key domain. Another study showed high risk of bias in two key domains. For one study, high risk of bias was found in three key domains. Another study showed high risk of bias in three key domains, as well as two unclear risks of bias in two key domains. A further study revealed two key domains with high risk of bias and two key domains with unclear risk of bias. According to 2011 definitions, ${ }^{12}$ the overall ranking revealed only two studies with a low risk of bias. All of the studies revealed a tendency of a high risk of bias, resulting in an overall unclear and high risk of bias across studies.

On the other hand, the scores of the 12 nonrandomized studies eligible for the Newcastle-Ottawa scale ranged 5-8, and the mean score was 6.9. For the other 13 studies assessed by den Hartog et al quality-assessment tool, ${ }^{14}$ all those included had a score of 5 or more. 
Table I Excluded articles and reasons for exclusion

\begin{tabular}{|c|c|}
\hline Study & Reason for exclusion \\
\hline Bonde et $\mathrm{al}^{15}$ & Did not allow data extraction \\
\hline Bressan et $\mathrm{al}^{16}$ & No follow-up \\
\hline Büchi et ll $^{17}$ & No follow-up \\
\hline Camargos et $\mathrm{al}^{18}$ & Did not allow data extraction \\
\hline Cooper et al ${ }^{19}$ & Did not allow data extraction \\
\hline Cosgarea et $\mathrm{al}^{20}$ & No follow-up \\
\hline Deporter et $\mathrm{al}^{21}$ & Did not allow data extraction \\
\hline Ferrari et $\mathrm{al}^{22}$ & Did not allow data extraction \\
\hline Happe et $\mathrm{al}^{23}$ & No follow-up \\
\hline Kreissl et $\mathrm{al}^{25}$ & Did not allow data extraction \\
\hline Jung and Yoon ${ }^{24}$ & Did not allow data extraction \\
\hline Payer et $\mathrm{al}^{26}$ & Single-piece implant \\
\hline Pettersson and Sennerby ${ }^{27}$ & Did not allow data extraction \\
\hline Redemagni et $\mathrm{al}^{28}$ & Did not allow data extraction \\
\hline Rompen et $\mathrm{al}^{29}$ & Did not allow data extraction \\
\hline van Brakel et $\mathrm{al}^{30}$ & No follow-up \\
\hline van Brakel et $\mathrm{al}^{31}$ & 3-month follow-up \\
\hline Vanlıoğlu et $\mathrm{al}^{32}$ & Did not allow data extraction \\
\hline Visser et $\mathrm{al}^{33}$ & Did not allow data extraction \\
\hline
\end{tabular}

\section{Included studies}

The patients in the included studies were treated in university (24 studies), private practice (five studies), and multicenter (one study) settings, while in two studies the setting was not reported. A total of 2,127 implants were placed in 1,529 patients aged 17-77 years, with a mean age of 42.1 years. The majority of studies (17) reported on anterior and posterior abutment, while eight reported on anterior abutment only and seven described posterior abutment only; 19 studies evaluated implant systems with internal implant-abutment connections, ten evaluated implant systems with external implant-abutment connection, and three reported on both internal and external implant-abutment connections. Almost all studies reported use of abutments to support single-crown restorations (29 studies), one study reported use of abutments to support fixed partial dentures, and two studies reported use of both. The majority of the studies (24) reported use of

Table 2 Characteristics of included studies

\begin{tabular}{|c|c|c|c|c|c|}
\hline Study & Type & Setting & Patients, n & Mean age, years & Mean follow-up, years \\
\hline de Albornoz et $\mathrm{al}^{34}$ & RCT & University & 26 & 51.7 & I \\
\hline Bae et $\mathrm{al}^{35}$ & Prospective & University & 19 & 47 & I \\
\hline Cabello et $\mathrm{al}^{36}$ & Prospective & Private & 14 & 52 & I \\
\hline Canullo and Götz ${ }^{37}$ & Prospective & NR & 5 & 49.1 & $\mathrm{I} .5$ \\
\hline Canullo ${ }^{38}$ & Prospective & Private & 25 & 52.28 & 3.3 \\
\hline Cionca et $\mathrm{al}^{39}$ & Prospective & University & 32 & 51.9 & 1.6 \\
\hline Cooper et $\mathrm{al}^{40}$ & Prospective & University & 41 & 30.6 & 3 \\
\hline Cosyn et $\mathrm{al}^{4 \mathrm{I}}$ & Prospective & University & 25 & 54 & 3 \\
\hline den Hartog et $\mathrm{al}^{42}$ & $\mathrm{RCT}$ & University & 62 & 39.3 & 1.5 \\
\hline Ekfeldt et $\mathrm{al}^{43}$ & Retrospective & Private & 130 & 23 & NR \\
\hline Furze et $\mathrm{al}^{44}$ & Prospective & Private & 10 & 45.1 & I \\
\hline Galluci et $\mathrm{al}^{45}$ & $\mathrm{RCT}$ & University & 20 & NR & 2 \\
\hline Gotfredsen ${ }^{46}$ & Prospective & University & 20 & 33 & 10 \\
\hline Guljé et $\mathrm{al}^{47}$ & Prospective & Multicenter & 21 & 57 & I \\
\hline Hosseini et $\mathrm{al}^{48}$ & $\mathrm{RCT}$ & University & 36 & 28.1 & I \\
\hline Hosseini et $\mathrm{al}^{49}$ & Prospective & University & 59 & 27.9 & 3 \\
\hline Jemt $t^{50}$ & Retrospective & University & 35 & 33.5 & 10 \\
\hline Kim et $\mathrm{al}^{51}$ & Prospective & University & 213 & 56.5 & 3.6 \\
\hline Lee and Hasegawa ${ }^{52}$ & Prospective & NR & 9 & 42 & I \\
\hline Lops et $\mathrm{al}^{53}$ & Prospective & University & 81 & 54 & 5 \\
\hline Lops et $\mathrm{al}^{54}$ & Prospective & University & 72 & 46 & 2 \\
\hline MacDonald et $\mathrm{al}^{55}$ & Prospective & University & 20 & 43.5 & NR \\
\hline Nejatidanesh et $\mathrm{al}^{56}$ & Retrospective & University & 122 & 50 & 4.9 \\
\hline Nothdurft and Pospiech ${ }^{57}$ & Prospective & University & 24 & NR & 1 \\
\hline Passos et $\mathrm{al}^{58}$ & Retrospective & University & $|4|$ & NR & NR \\
\hline Payer et $\mathrm{al}^{59}$ & $\mathrm{RCT}$ & University & 30 & NR & 2 \\
\hline Pozzi et $\mathrm{al}^{60}$ & Prospective & University & 27 & 54.18 & 3.6 \\
\hline Vanlıoglu et $\mathrm{al}^{61}$ & Prospective & University & 12 & 33.2 & 5 \\
\hline Vigolo et $\mathrm{al}^{62}$ & $\mathrm{RCT}$ & University & 20 & NR & 4 \\
\hline Vigolo and Givani63 & Prospective & Private & 144 & 37 & 5 \\
\hline Zembic et $\mathrm{al}^{64}$ & $\mathrm{RCT}$ & University & 18 & 41.6 & 5.6 \\
\hline Zembic et $\mathrm{al}^{65}$ & Prospective & University & 16 & 46 & 11.3 \\
\hline
\end{tabular}

Abbreviations: RCT, randomized controlled trial; NR, not reported. 
Table 3 Risk of bias for randomized controlled trials

\begin{tabular}{|c|c|c|c|c|c|c|c|}
\hline Study & $\begin{array}{l}\text { Random- } \\
\text { sequence } \\
\text { generation }\end{array}$ & $\begin{array}{l}\text { Allocation } \\
\text { concealment }\end{array}$ & $\begin{array}{l}\text { Blinding } \\
\text { (participants } \\
\text { and personnel) }\end{array}$ & $\begin{array}{l}\text { Blinding } \\
\text { (outcome } \\
\text { assessment) }\end{array}$ & $\begin{array}{l}\text { Incomplete } \\
\text { outcome data } \\
\text { addressed }\end{array}$ & $\begin{array}{l}\text { Selective } \\
\text { reporting }\end{array}$ & $\begin{array}{l}\text { Other } \\
\text { bias }\end{array}$ \\
\hline De Albornoz et $\mathrm{al}^{34}$ & Low & Low & Low & Low & Low & Unclear & No \\
\hline den Hartog et $\mathrm{al}^{42}$ & Low & Low & Low & Low & Low & Low & No \\
\hline Galluci et $\mathrm{al}^{45}$ & Low & Low & Low & Low & Low & Low & No \\
\hline Hosseini et $\mathrm{al}^{48}$ & High & Low & Low & Low & High & Low & No \\
\hline Payer et $\mathrm{al}^{59}$ & Low & Low & High & High & High & Low & No \\
\hline Vigolo et $\mathrm{al}^{62}$ & Low & Unclear & High & High & Low & Unclear & No \\
\hline Zembic et $\mathrm{al}^{64}$ & Low & Unclear & Unclear & High & High & High & No \\
\hline
\end{tabular}

Table 4 Quality of included studies using Newcastle-Ottawa scale

\begin{tabular}{|c|c|c|c|c|}
\hline Study & Selection $* * * *$ & Comparability** & Outcome ${ }^{* * *}$ & Score \\
\hline Canullo ${ }^{38}$ & $* * * *$ & $*$ & * & 6 \\
\hline Gotfredsen ${ }^{46}$ & $* * * *$ & $*$ & $* *$ & 7 \\
\hline Hosseini et $\mathrm{al}^{49}$ & $* * * *$ & $*$ & $* * *$ & 8 \\
\hline Jemt $t^{50}$ & $* * * *$ & $*$ & $* * *$ & 8 \\
\hline Kim et $\mathrm{al}^{51}$ & $* *$ & $*$ & $* *$ & 5 \\
\hline Lops et $\mathrm{a}^{53}$ & $* * * *$ & $*$ & $* * *$ & 8 \\
\hline Lops et $\mathrm{a}^{54}$ & $* * * *$ & $*$ & $* *$ & 7 \\
\hline Nejatidanesh et $\mathrm{al}^{56}$ & $* * *$ & $*$ & $* * *$ & 7 \\
\hline Passos et $a^{58}$ & $* *$ & $*$ & $* * *$ & 6 \\
\hline Pozzi et a ${ }^{60}$ & $* *$ & $* *$ & $* * *$ & 7 \\
\hline Vanlıoglu et $a^{\mid 61}$ & $* * * *$ & $*$ & $* *$ & 7 \\
\hline Vigolo and Givani ${ }^{63}$ & $* * * *$ & $*$ & $* *$ & 7 \\
\hline
\end{tabular}

Note: Each * refers to the number of points. Selection of the study groups (up to 4 points), comparability of the groups (up to 2 points), and exposure or outcome (up to 3 points)..

cement-retained restorations. Screw-retained restorations were reported exclusively in eight studies, and six studies reported the use of both cement- and screw-retained restorations. For definitive crown material fabricated over the abutments, 16 studies reported on the use of all-ceramic crown, seven on metal ceramic crowns, eight on both all-ceramic and metal ceramic crowns, and one did not mention the type of crown used (Table 5).

The 32 studies included a total number of 2,127 implants placed in 1,529 patients, with follow-up of 1-12 (mean 3.3) years. A total of 863 implants were placed in the anterior region and 1,264 implants in the posterior region. Altogether, 1,242 zirconia abutments, 646 titanium abutments, and 239 UCLA abutments were evaluated at follow-up in the included studies. Of the total number of zirconia abutments, 623 zirconia abutments were used in the anterior region and 619 in the posterior region. Titanium abutments were divided into 214 abutments in the anterior region and 432 in the posterior region. There were 26 UCLA abutments in the anterior region and 213 in the posterior region.

\section{Implant abutments in the anterior region}

A total number of 863 abutments were placed in the anterior region, of which 623 were zirconia abutment, 214 titanium abutments, and 26 UCLA abutments. Abutment failure occurred in ten abutments, all of which were zirconia. The failure manifested as abutment fracture. No abutment failure was reported in titanium or UCLA abutments.

One implant restored with a titanium abutment was lost at the 1-month follow-up due to mobility. Only one implant supporting a titanium abutment was lost after loading in the anterior region. Nine technical complications were reported: six related to zirconia abutments and three related to titanium abutments. All the nine technical complications were abutment-screw loosening. A total of 34 prosthetic complications were reported: 19 related to zirconia abutments, 14 related to titanium abutments, and 1 related to a UCLA abutment. Prosthetic complications were minor chipping of porcelain (17), loss of crown retention (13), and major complications leading to crown remake (four), such as major chipping of porcelain and unacceptable marginal adaptation. There were 16 biological complications reported: nine related to zirconia abutments, five to titanium abutments, and two to UCLA abutments. Biological complications were recession (eight), buccal fistulas (six), peri-implant bone loss $>2 \mathrm{~mm}$ (one), and peri-implant mucosal defect (one). There were no aesthetic complications reported, although two studies reported better aesthetic outcomes for zirconia abutments. ${ }^{34,49}$ 
Table 5 Abutment material and prosthetic characteristics

\begin{tabular}{|c|c|c|c|c|c|c|}
\hline Study & $\begin{array}{l}\text { Type of } \\
\text { implant } \\
\text { restoration }\end{array}$ & $\begin{array}{l}\text { Type of } \\
\text { abutment } \\
\text { connection }\end{array}$ & $\begin{array}{l}\text { Type of abutment } \\
\text { material }\end{array}$ & $\begin{array}{l}\text { Abutment } \\
\text { manufacturer }\end{array}$ & $\begin{array}{l}\text { Prosthetic } \\
\text { restoration } \\
\text { material }\end{array}$ & $\begin{array}{l}\text { Nature of } \\
\text { prosthetic } \\
\text { restoration }\end{array}$ \\
\hline $\begin{array}{l}\text { de Albornoz } \\
\text { et al }\left.\right|^{34}\end{array}$ & Single crown & Internal & $\begin{array}{l}\text { Prefabricated } \mathrm{Zr} \text { and } \mathrm{Ti} \\
\text { abutments }\end{array}$ & Ti/SPI Easy-Zr/Spy Art & $A C$ & Cement retained \\
\hline Bae et $\mathrm{al}^{35}$ & $\begin{array}{l}\text { Single crown } \\
\text { and FPDs }\end{array}$ & External & $\begin{array}{l}\text { Prefabricated alumina- } \\
\text { toughened } \mathrm{Zr} \text { abutment }\end{array}$ & ZirAce, Acucera & $A C$ & Cement retained \\
\hline Cabello et $\mathrm{al}^{36}$ & Single crown & Internal & $\begin{array}{l}\text { UCLA and prefabricated } \mathrm{Zr} \\
\text { and custom-made } \mathrm{Zr}\end{array}$ & Straumann & $M C, A C$ & $\begin{array}{l}\text { Screw retained } \\
(12) \text { and cement } \\
\text { retained ( } 2)\end{array}$ \\
\hline $\begin{array}{l}\text { Canullo and } \\
\text { Götz }^{37}\end{array}$ & Single crown & Internal & Prefabricated $\mathrm{Ti}$ & Sweden and Martina & MC & Cement retained \\
\hline Canullo ${ }^{38}$ & Single crown & Internal & $\begin{array}{l}\text { CadCam } \mathrm{Zr} \text { on a } \\
\text { prefabricated Ti base }\end{array}$ & $\begin{array}{l}\text { ProUnic abutment, } \\
\text { implanted with } \\
\text { Zirkonzahn }\end{array}$ & $A C$ & Cement retained \\
\hline Cionca et $\mathrm{al}^{39}$ & Single crown & Internal & Prefabricated Zr & $\begin{array}{l}\text { Zeramex T implant } \\
\text { system; Dental Point }\end{array}$ & $A C$ & Cement retained \\
\hline Cooper et al ${ }^{40}$ & Single crown & Internal & Prefabricated $\mathrm{Ti}$ & $\begin{array}{l}\text { Astra abutment ST } \\
\text { titanium }\end{array}$ & NR & Cement retained \\
\hline Cosyn et $\mathrm{al}^{41}$ & Single crown & Internal & Prefabricated $\mathrm{Ti}$ & $\begin{array}{l}\text { Aesthetic abutment, } \\
\text { Nobel Biocare }\end{array}$ & MC & Cement retained \\
\hline $\begin{array}{l}\text { den Hartog } \\
\text { et a }{ }^{42}\end{array}$ & Single crown & Internal & Individually fabricated $\mathrm{Zr}$ & Procera, Nobel Biocare & $A C$ & $\begin{array}{l}\text { Cement and screw } \\
\text { retained }\end{array}$ \\
\hline Ekfeldt et al ${ }^{43}$ & Single crown & $\begin{array}{l}\text { Internal and } \\
\text { External }\end{array}$ & CadCam Zr & Procera, Nobel Biocare & $A C$ & $\begin{array}{l}\text { One-piece screw } \\
\text { and cement } \\
\text { retained }\end{array}$ \\
\hline Furze et a $\mathrm{a}^{44}$ & Single crown & Internal & CadCam Zr & Straumann & $A C$ & Cement retained \\
\hline Galluci et al ${ }^{45}$ & Single crown & Internal & $\begin{array}{l}\text { Prefabricated Ti coupled with } \\
\text { In-Ceram alumina or cast- } \\
\text { gold alloy }\end{array}$ & $\begin{array}{l}\text { SynOcta I.5 screw- } \\
\text { retained abutment, } \\
\text { Straumann }\end{array}$ & $M C, A C$ & $\begin{array}{l}\text { Two-piece screw } \\
\text { retained }\end{array}$ \\
\hline Gotfredsen $^{46}$ & Single crown & Internal & $\begin{array}{l}\text { Prefabricated and custom- } \\
\text { made } \mathrm{Ti}\end{array}$ & $\begin{array}{l}\text { Astra abutment } \\
\text { ST titanium, Astra } \\
\text { preparable abutments }\end{array}$ & MC & Cement retained \\
\hline Guljé et a $\mathrm{a}^{47}$ & Single crown & External & $\begin{array}{l}\text { Custom-made titanium } \\
\text { abutments }\end{array}$ & Atlantis, Dentsply & $A C$ & Cement retained \\
\hline Hosseini et a $\left.\right|^{48}$ & Single crown & Internal & $\begin{array}{l}\text { Prefabricated } \mathrm{Zr} \text { and } \mathrm{Ti} \text { and } \\
\text { gold abutments }\end{array}$ & $\begin{array}{l}\text { ZirDesign (Astra Tech), } \\
\text { Ti Design (Astra Tech), } \\
\text { Cast-To (Astra Tech) }\end{array}$ & MC,AC & Cement retained \\
\hline Hosseini et $\mathrm{al}^{49}$ & Single crown & Internal & $\begin{array}{l}\text { UCLA-prefabricated } \mathrm{Zr} \text { - } \\
\text { prefabricated } \mathrm{Ti}\end{array}$ & Astra Tech & $M C, A C$ & Cement retained \\
\hline Jemt $t^{50}$ & Single crown & External & Prefabricated titanium & $\begin{array}{l}\text { TiAdapt and CeraOne, } \\
\text { Nobel Biocare }\end{array}$ & $M C$ & $\begin{array}{l}\text { One-piece screw } \\
\text { retained and } \\
\text { externally cemented } \\
\text { crowns with single- } \\
\text { abutment screw }\end{array}$ \\
\hline Kim et $\mathrm{al}^{51}$ & $\begin{array}{l}\text { Single crown } \\
\text { and FPDs }\end{array}$ & External & $\begin{array}{l}\text { Prefabricated alumina- } \\
\text { toughened } \mathrm{Zr} \text { abutment }\end{array}$ & ZirAce, Acucera & $M C, A C$ & $\begin{array}{l}\text { One-piece screw } \\
\text { and cement } \\
\text { retained }\end{array}$ \\
\hline $\begin{array}{l}\text { Lee and } \\
\text { Hasegawa }^{52}\end{array}$ & Single crown & Internal & $\begin{array}{l}\text { Prefabricated } \mathrm{Zr} \text { with a } \mathrm{Ti} \\
\text { interface ring }\end{array}$ & $\begin{array}{l}\text { Zimmer contour all } \\
\text { ceramic abutment }\end{array}$ & $A C$ & Cement retained \\
\hline Lops et $\mathrm{al}^{53}$ & Single crown & Internal & $\begin{array}{l}\text { Prefabricated } \mathrm{Zr} \text { and } \mathrm{Ti} \\
\text { abutments }\end{array}$ & $\begin{array}{l}\text { Ti/profile bi abutment, } \\
\text { Astra Tech Zr/ceramic } \\
\text { abutment ST ZirDesign } \\
\text { abutment, Astra Tech }\end{array}$ & $M C, A C$ & Cement retained \\
\hline Lops et $\mathrm{al}^{54}$ & Single crown & External & $\begin{array}{l}\text { Prefabricated } \mathrm{Zr} \text { and } \mathrm{Ti} \text {, } \\
\text { CadCam } \mathrm{Zr} \text { and } \mathrm{Ti}\end{array}$ & $\begin{array}{l}\text { ZirDesign (Astra Tech), } \\
\text { TiDesign (Astra Tech), } \\
\text { Zr Atlantis, Ti Atlantis }\end{array}$ & $\mathrm{MC}, \mathrm{AC}$ & Cement retained \\
\hline
\end{tabular}


Table 5 (Continued)

\begin{tabular}{|c|c|c|c|c|c|c|}
\hline Study & $\begin{array}{l}\text { Type of } \\
\text { implant } \\
\text { restoration }\end{array}$ & $\begin{array}{l}\text { Type of } \\
\text { abutment } \\
\text { connection }\end{array}$ & $\begin{array}{l}\text { Type of abutment } \\
\text { material }\end{array}$ & $\begin{array}{l}\text { Abutment } \\
\text { manufacturer }\end{array}$ & $\begin{array}{l}\text { Prosthetic } \\
\text { restoration } \\
\text { material }\end{array}$ & $\begin{array}{l}\text { Nature of } \\
\text { prosthetic } \\
\text { restoration }\end{array}$ \\
\hline $\begin{array}{l}\text { MacDonald } \\
\text { et } \mathrm{al}^{55}\end{array}$ & Single crown & External & Prefabricated $\mathrm{Ti}$ & Not reported & MC & Screw retained \\
\hline $\begin{array}{l}\text { Nejatidanesh } \\
\text { et } \mathrm{al}^{56}\end{array}$ & Single crown & Internal & Prefabricated $\mathrm{Ti}$ & SynOcta, Straumann & $A C$ & Cement retained \\
\hline $\begin{array}{l}\text { Nothdurft and } \\
\text { Pospiech }^{57}\end{array}$ & Single crown & Internal & Prefabricated $\mathrm{Zr}$ & $\begin{array}{l}\text { Cercon abutment, } \\
\text { Dentsply Friadent }\end{array}$ & $A C$ & Cement retained \\
\hline Passos et $\mathrm{al}^{58}$ & Single crown & $\begin{array}{l}\text { Internal and } \\
\text { External }\end{array}$ & $\begin{array}{l}\text { Prefabricated and customized } \\
\mathrm{Zr}\end{array}$ & $\begin{array}{l}\text { 3i, Astra Tech, Nobel } \\
\text { Biocare, Straumann }\end{array}$ & $A C$ & Cement retained \\
\hline Payer et $\mathrm{al}^{59}$ & Single crown & Internal & $\begin{array}{l}\text { Prefabricated } \mathrm{Zr} \text { and } \mathrm{Ti} \\
\text { abutments }\end{array}$ & $\begin{array}{l}\text { Ziteron } \mathrm{Zr} \text { abutment, } \\
\text { Ziteron } \mathrm{Ti} \text { abutment }\end{array}$ & $A C$ & Cement retained \\
\hline Pozzi et al ${ }^{60}$ & FPD & $\begin{array}{l}\text { Internal and } \\
\text { External }\end{array}$ & CadCam $\mathrm{Zr}$ and $\mathrm{Ti}$ & Procera, Nobel Biocare & $A C$ & Cement retained \\
\hline Vanlıoglu et $\mathrm{al}^{61}$ & Single crowns & Internal & $\begin{array}{l}\text { Prefabricated } \mathrm{Ti} \text { and } \\
\text { custom-made } \mathrm{Zr} \text { MAD/MAM } \\
\text { (Zirkonzhan, Steger) }\end{array}$ & Not reported & $A C$ & Cement retained \\
\hline Vigolo et $\mathrm{al}^{62}$ & Single crown & External & Titanium and gold UCLA & $\begin{array}{l}\text { Ti, Procera, Nobel } \\
\text { Biocare, gold, SGUCAIC, } \\
\text { 3i, Implant Innovations }\end{array}$ & $\mathrm{MC}$ & Cement retained \\
\hline $\begin{array}{l}\text { Vigolo and } \\
\text { Givani }{ }^{63}\end{array}$ & Single crown & External & Custom-made gold UCLA & $\begin{array}{l}\text { SWGA5IC, SGUCAIC, } \\
\text { 3i, Implant Innovations }\end{array}$ & $\mathrm{MC}$ & Cement retained \\
\hline Zembic et $\mathrm{al}^{64}$ & Single crown & External & CadCam $\mathrm{Ti}$ and CadCam $\mathrm{Zr}$ & Procera, Nobel Biocare & $\mathrm{MC}, \mathrm{AC}$ & $\begin{array}{l}\text { Cement and two } \\
\text { screws retained }\end{array}$ \\
\hline Zembic et al ${ }^{65}$ & Single crown & External & $\begin{array}{l}\text { Customized experimental } \mathrm{Zr} \\
\text { abutments }\end{array}$ & Wohlwend & $A C$ & Cement retained \\
\hline
\end{tabular}

Abbreviations: MC, metal ceramic; AC, all ceramic; FPDs, fixed partial dentures.

In general, studies showed minimal differences in aesthetic outcomes and patient satisfaction when comparing ceramic and metal abutments (Table 6).

\section{Implant abutments in the posterior region}

Altogether, 1,264 abutments were placed in the posterior region: 619 zirconia, 432 titanium, and 213 UCLA. Failure occurred in nine of the zirconia abutments, manifesting as abutment fracture. No abutment failures were reported in titanium or UCLA abutments (Tables 6 and 7). A total of 14 implants were lost due to loss of osseointegration: ten were supporting zirconia abutments and four supporting titanium abutments. A total of 27 technical complications were reported, the majority of which occurred in zirconia abutments (25 of 27), while two occurred in titanium abutments. All technical complications were abutment-screw loosening, except one, a screw fracture that occurred in an externalconnection zirconia abutment. A total of 32 prosthetic complications were reported: eleven related to zirconia-supported crowns and 21 to metal-supported crowns. The majority of prosthetic complications were minor chipping of porcelain
(29); the other complication was loss of retention, one of which needed to be remade. There were eleven biological complications: four related to zirconia abutments and seven to metal abutments. Six of the complications were suppuration on probing and pocket depth $>5 \mathrm{~mm}$, four were buccal marginal fistulas, and one was implant mucositis (Table 6).

\section{Meta-analysis}

Due to heterogeneity of study designs and reported data, only within-study comparison of failure events of anterior and posterior zirconia-implant abutments was possible, and this was feasible in only five studies involving 660 implants (one RCT, three prospective, one retrospective), which is illustrated as a forest plot in Figure 2. Based on the fixedeffect model, no significant difference in failure rates were found between anterior and posterior zirconia abutments (RR $1.53,95 \%$ CI $0.49-4.77 ; P=0.47)$. No within-study $\left(\chi^{2}=2.09\right.$, $P=0.72)$ or between-study $\left(I^{2}=0\right)$ heterogeneity was observed.

\section{Publication bias}

Visual examination of the funnel plot indicated low-level publication bias evident from the symmetrical distribution for all studies (Figure 3). 


\section{Discussion}

Systematic reviews are often useful in the evaluation of different materials, since they extract the best evidence from the scientific literature; therefore, this systematic review and meta-analysis was conducted to compare the clinical outcome of different abutment materials on abutment-failure rate and technical, prosthetic, biological, and aesthetic outcomes in anterior and posterior regions. Due to heterogeneity of the included studies, variation of data included, and outcome results, a meta-analysis was feasible only for zirconiaabutment failure in anterior and posterior regions. All the reported abutment failures manifested as fractures. The results of the meta-analysis showed that implant-abutment failure did not seem to be affected by position in the jaw. Zirconia abutments exhibited similar fracture rates in anterior and posterior regions. On the other hand, no titanium abutments fractured. Usually, fractures of metal abutments are scarce. This is in accordance with another systematic review that indicated a fracture rate of $0.07 \%$ at 5 years. ${ }^{8}$ The majority of data available endorsing zirconia abutment loading refer to stimulated treatments of anterior teeth. ${ }^{66-69}$ These in vitro studies suggested that zirconia abutments were suitable to withstand occlusal loading for anterior sites in normal human subjects with fractures at loads above $400 \mathrm{~N} .{ }^{70}$ On the other hand, in vitro studies involving ceramic-implant abutments with a focus on stimulated treatments of posterior teeth were not found in the published literature.

A previous systematic review showed that failures in the anterior region were restricted to ceramic abutments, ${ }^{10}$ but this is in contrast to other systematic reviews reporting no differences in the survival and failure rates of ceramic and metal abutments. ${ }^{8,9,11}$ Therefore, superior clinical behavior for zirconia might be expected, and it might even serve as an alternative to metal in various indications.

Our study showed significant differences in implantfailure rates in anterior and posterior regions $(0.1 \%$ anterior, $1.1 \%$ posterior), all of which were lost after loading. In the posterior region, $1.6 \%$ of implants supporting zirconia abutments and $0.9 \%$ of implants supporting titanium abutments failed. Failure reasons ranged from loss of osseointegration, marginal bone loss $\geq 2 \mathrm{~mm}$, and aseptic loosening. Long-term implant-survival studies have even indicated that the posterior maxilla presents the lowest survival rate. ${ }^{71,72}$ One of the studies identified in this systematic review described the use of two-piece zirconia abutments, which contributed to five of the 14 implants lost in the posterior region. ${ }^{39}$

Technical complications were detected primarily in posterior regions, reflecting the high functional loading in this region. Complications were mostly observed in zirconia abutments. Abutment-screw loosening was the most common technical complication, accounting for all but one (abutmentscrew fracture) of the total complications. This is in accordance with previous systematic reviews. ${ }^{8-10}$ The incidence of screw loosening was minimal across the included studies, except for one,${ }^{51}$ which accounted for 23 of the total screwloosening events. In that study, alumina-toughened zirconia abutments were used.

Prosthetic complications showed no significant differences in anterior and posterior regions, regardless of abutment material used. The most common complications were minor chipping of porcelain and loss of crown retention, probably due to provisional cementation. Biological complications reported in the anterior region were buccal fistulas and gingival recessions, while in the posterior region only buccal fistulas were reported. A reason for this may be the increased risk of recession of thin gingiva in the anterior region compared to thicker gingiva in the posterior region. ${ }^{8}$ Biological outcomes did not reveal any differences between different abutment materials. This is in accordance with a systematic review ${ }^{8}$ and an animal study, ${ }^{73}$ which exhibited similar soft-tissue integration of different abutment materials. Only one of the included studies reported on probing depth $\geq 5 \mathrm{~mm}$ and/or suppuration affecting three implants supporting zirconia abutments in the posterior region. ${ }^{48}$ No aesthetic complications were reported in any of the included studies.

There was diversity in methods of assessment and measurements of aesthetic outcomes. Overall, no significant differences were found between zirconia and titanium abutments. The results of our study are in accordance with another systematic review, ${ }^{10}$ but in contrast to two other systematic reviews, ${ }^{8,74}$ which demonstrated superiority of ceramic abutments in terms of aesthetic outcome.

It is widely accepted that RCTs provide "gold standard" evidence of the effectiveness of therapies. However, probably due to costs associated with this type of research and due to ethical reasons, there is a scarcity of RCTs in implant research. Nonetheless, relevant information is not provided exclusively by RCTs. Cohort studies, case series, and clinical trials can still offer valuable longitudinal information. As such, those types of studies were considered for evaluation too.

A total of 13 studies included for this review could be classified as case series, and consequently were of a lower level of evidence. Although these studies were acceptable methodologically within their framework and well documented, results of these studies require cautious 
Table 6 Comparison of clinical outcomes in anterior and posterior regions

\begin{tabular}{|c|c|c|c|c|}
\hline \multirow[t]{2}{*}{ Study } & \multicolumn{4}{|l|}{ Anterior } \\
\hline & $\begin{array}{l}\text { Technical } \\
\text { complications }\end{array}$ & Prosthetic complications & Biological complications & $\begin{array}{l}\text { Aesthetic } \\
\text { complications }\end{array}$ \\
\hline de Albornoz et al $\left.\right|^{34}$ & None & None & None & None \\
\hline Bae et $\mathrm{al}^{35}$ & None & None & None & NR \\
\hline Cabello et $\mathrm{al}^{36}$ & None & $\begin{array}{l}\text { I loss of retention of crown } \\
\text { (abutment material not reported) }\end{array}$ & None & None \\
\hline Canullo and Götz ${ }^{37}$ & NR & NR & None & NR \\
\hline Canullo 38 & None & NA & None & NR \\
\hline Cionca et $\mathrm{al}^{39}$ & None & NA & None & None \\
\hline Cooper et $\mathrm{al}^{40}$ & None & $\begin{array}{l}3 \text { minor chipping of porcelain }(\mathrm{Zr}) \text {, } \\
\text { loss of retention of } 2 \text { crowns }(\mathrm{Zr})\end{array}$ & $\begin{array}{l}\text { Tenderness of buccal mucosa } \\
\text { and peri-implant mucosal defect } \\
\text { (I Zr) }\end{array}$ & NR \\
\hline Cosyn et al ${ }^{41}$ & $\begin{array}{l}\text { I implant lost due to } \\
\text { mobility }(\mathrm{Ti})\end{array}$ & $\begin{array}{l}\text { Loss of retention of I MC crown } \\
(\mathrm{Ti})\end{array}$ & $\begin{array}{l}\text { Mid-facial recession of mucosa } \\
(\mathrm{I} \mathrm{Ti})\end{array}$ & None \\
\hline den Hartog et $\mathrm{al}^{42}$ & None & None & None & None \\
\hline Ekfeldt et $\mathrm{al}^{43}$ & $\begin{array}{l}\text { I abutment screw } \\
\text { loosening }(\mathrm{Zr})\end{array}$ & 3 minor chipping of porcelain $(\mathrm{Zr})$ & None & None \\
\hline Furze et al ${ }^{14}$ & None & None & None & None \\
\hline Galluci et al ${ }^{45}$ & None & 2 minor chipping of porcelain $(2 \mathrm{Ti})$ & None & None \\
\hline Gotfredsen ${ }^{46}$ & $\begin{array}{l}2 \text { abutment-screw } \\
\text { loosening }(\mathrm{Ti})\end{array}$ & $\begin{array}{l}2 \text { crowns remade }(\mathrm{Ti}), \mathrm{I} \text { due to } \\
\text { abutment loosening and I due to } \\
\text { major ceramic fracture }\end{array}$ & I buccal fistula (Ti) & NR \\
\hline Guljé et a $\left.\right|^{47}$ & - & - & - & \\
\hline Hosseini et $\mathrm{al}^{48}$ & - & - & - & - \\
\hline Hosseini et $\mathrm{al}^{49}$ & None & $\begin{array}{l}\text { I major chipping of porcelain } \\
\text { (remade) }(\mathrm{Zr}) \text {, loss of retention of } \\
\text { I MC crown (Ti), I unacceptable } \\
\text { marginal adaptation (remade), I } \\
\text { UCLA }\end{array}$ & $\begin{array}{l}5 \text { buccal fistulas }(3 \mathrm{Zr}+2 \\
\text { UCLA) }\end{array}$ & None \\
\hline Jemt $t^{50}$ & NA & None & $\begin{array}{l}\text { I buccal fistula }(\mathrm{Ti}) \\
2 \text { gingival recession }(\mathrm{Ti})\end{array}$ & NR \\
\hline Kim et $\mathrm{al}^{51}$ & $\begin{array}{l}\text { I abutment-screw } \\
\text { loosening }(\mathrm{Zr})\end{array}$ & NR & None & NR \\
\hline Lee and Hasegawa ${ }^{52}$ & None & None & None & None \\
\hline Lops et $\mathrm{a}^{53}$ & - & - & - & - \\
\hline Lops et $\mathrm{al}^{54}$ & $\begin{array}{l}\text { I abutment-screw } \\
\text { loosening }(Z r)\end{array}$ & None & None & NR \\
\hline MacDonald et al ${ }^{55}$ & $\begin{array}{l}\text { I abutment-screw } \\
\text { loosening }(\mathrm{Ti})\end{array}$ & None & None & NR \\
\hline Nejatidanesh et al ${ }^{56}$ & None & 7 minor chipping of porcelain $(\mathrm{Ti})$ & NA & None \\
\hline $\begin{array}{l}\text { Nothdurft and } \\
\text { Pospiech }^{57}\end{array}$ & - & - & - & - \\
\hline
\end{tabular}




\begin{tabular}{|c|c|c|c|c|}
\hline \multicolumn{4}{|l|}{ Posterior } & \multirow{2}{*}{$\begin{array}{l}\text { Other } \\
\text { Notes }\end{array}$} \\
\hline $\begin{array}{l}\text { Technical } \\
\text { complications }\end{array}$ & Prosthetic complications & Biological complications & $\begin{array}{l}\text { Aesthetic } \\
\text { complications }\end{array}$ & \\
\hline None & None & None & None & \\
\hline None & None & None & NR & \\
\hline- & - & - & - & \\
\hline - & - & - & - & \\
\hline None & NA & None & NR & $\begin{array}{l}\text { I minor chipping of porcelain } \\
(\mathrm{Zr}) \text {, site not reported }\end{array}$ \\
\hline $\begin{array}{l}5 \text { implants lost due to } \\
\text { aseptic loosening ( } \mathrm{Zr} \text { ) }\end{array}$ & NA & None & None & $\begin{array}{l}\text { I minor chipping of porcelain } \\
(\mathrm{Zr}) \text {, site not reported }\end{array}$ \\
\hline- & - & - & - & \\
\hline- & - & - & - & \\
\hline None & None & None & None & \\
\hline None & None & None & None & \\
\hline None & None & None & None & \\
\hline- & - & - & - & \\
\hline NA & NA & None & NR & $\begin{array}{l}2 \text { minor porcelain fracture }(\mathrm{Ti}) \text {, } \\
\text { position not reported } \\
2 \text { crown loosening }(\mathrm{Ti}) \text {, position } \\
\text { not reported } \\
\text { I implant } \geq 2 \mathrm{~mm} \text { bone loss }(\mathrm{Ti}) \text {, } \\
\text { position not reported }\end{array}$ \\
\hline None & None & None & None & \\
\hline None & $\begin{array}{l}\text { I minor chipping of porcelain } \\
\text { (abutment material not } \\
\text { reported) } \\
\text { Loss of retention of I MC } \\
\text { crown (remade) (abutment } \\
\text { material not reported) }\end{array}$ & $\begin{array}{l}\text { I buccal fistula }(\mathrm{Zr}) \\
\text { I suppuration on probing }(\mathrm{Zr}) \\
2 \text { pocket depth } \geq 5 \mathrm{~mm}(\mathrm{Zr}) \\
3 \text { suppuration on probing and } \\
\text { pocket depth } \geq 5 \mathrm{~mm} \text { (abutment } \\
\text { material not reported) }\end{array}$ & None & \\
\hline None & $\begin{array}{l}\text { Loss of retention of } 2 \mathrm{MC} \\
\text { crowns }(\mathrm{Ti})\end{array}$ & None & None & \\
\hline NA & None & 3 buccal fistulas $(\mathrm{Ti})$ & NR & $\begin{array}{l}5 \text { screw loosening }(\mathrm{Ti}) \text {, location } \\
\text { not reported }\end{array}$ \\
\hline $\begin{array}{l}23 \text { abutment-screw } \\
\text { loosening }(Z r), I \\
\text { abutment-screw fracture } \\
(Z r)\end{array}$ & NR & None & NR & \\
\hline- & - & - & - & \\
\hline $\begin{array}{l}2 \text { screw loosening ( } \mathrm{Zr} \text {, } \\
\mathrm{I} \mathrm{Ti})\end{array}$ & $\begin{array}{l}7 \text { minor chipping of porcelain } \\
(4 \mathrm{Zr}, 3 \mathrm{Ti})\end{array}$ & $\begin{array}{l}\text { Mucositis of implant supporting } \\
\text { metal crown }\left(T_{i}\right)\end{array}$ & NR & \\
\hline $\begin{array}{l}\text { I abutment-screw } \\
\text { loosening }(\mathrm{Ti})\end{array}$ & None & None & NR & \\
\hline $\begin{array}{l}\text { I implant lost due to } \\
\text { marginal bone loss } \\
\leq 2 \mathrm{~mm}(\mathrm{Zr})\end{array}$ & None & None & NR & $\begin{array}{l}\text { I abutment-screw loosening (Ti), } \\
\text { location not reported }\end{array}$ \\
\hline $\begin{array}{l}\text { I implant lost due to loss } \\
\text { of osseointegration (Ti) }\end{array}$ & $\begin{array}{l}12 \text { minor chipping of } \\
\text { porcelain }(\mathrm{Ti})\end{array}$ & NA & None & $\begin{array}{l}\text { I4 implants had pocket depth } \geq 4 \\
\mathrm{~mm}(\mathrm{Ti}) \text { location not reported }\end{array}$ \\
\hline None & $\begin{array}{l}4 \text { minor chipping of porcelain } \\
(\mathrm{Zr})\end{array}$ & None & NR & \\
\hline
\end{tabular}

(Continued) 
Table 6 (Continued)

\begin{tabular}{|c|c|c|c|c|}
\hline \multirow[t]{2}{*}{ Study } & \multicolumn{4}{|l|}{ Anterior } \\
\hline & $\begin{array}{l}\text { Technical } \\
\text { complications }\end{array}$ & Prosthetic complications & Biological complications & $\begin{array}{l}\text { Aesthetic } \\
\text { complications }\end{array}$ \\
\hline Passos et $\mathrm{al}^{58}$ & $\begin{array}{l}\text { I abutment-screw } \\
\text { loosening }(\mathrm{Zr})\end{array}$ & 7 crown decementation $(\mathrm{Zr})$ & 5 gingival recession $\geq 2(\mathrm{Zr})$ & NR \\
\hline Payer et $\mathrm{al}^{59}$ & None & None & None & None \\
\hline Pozzi et $\mathrm{al}^{60}$ & - & - & - & - \\
\hline Vanlıoglu et $a^{61}$ & $\begin{array}{l}\text { I abutment-screw } \\
\text { loosening (abutment } \\
\text { material not reported) }\end{array}$ & None & None & None \\
\hline Vigolo et $\mathrm{al}^{62}$ & - & - & - & - \\
\hline Vigolo and Givani ${ }^{63}$ & - & - & - & - \\
\hline Zembic et al ${ }^{64}$ & None & I minor chipping of porcelain $(\mathrm{Ti})$ & None & None \\
\hline Zembic et al ${ }^{65}$ & 2 screw loosening $(Z r)$ & 3 minor chipping of porcelain $(\mathrm{Zr})$ & None & NR \\
\hline Total & $\begin{array}{l}\text { I implant lost }(\mathrm{Ti}) / 6 \\
\text { abutment loosening }(\mathrm{Zr}) / 3 \\
\text { abutment loosening }(\mathrm{Ti})\end{array}$ & $\begin{array}{l}\text { I } 2 \text { loss of crown retention/19 minor } \\
\text { chipping/two major ceramic fracture/ } \\
\text { one unacceptable margin }\end{array}$ & $\begin{array}{l}7 \text { fistulas }(3 \mathrm{Zr}, 2 \mathrm{Ti}, 2 \mathrm{UCLA}) / 8 \\
\text { recession }(5 \mathrm{Zr}, 3 \mathrm{Ti})\end{array}$ & \\
\hline
\end{tabular}

Abbreviations: NA, not available; NR, not reported.

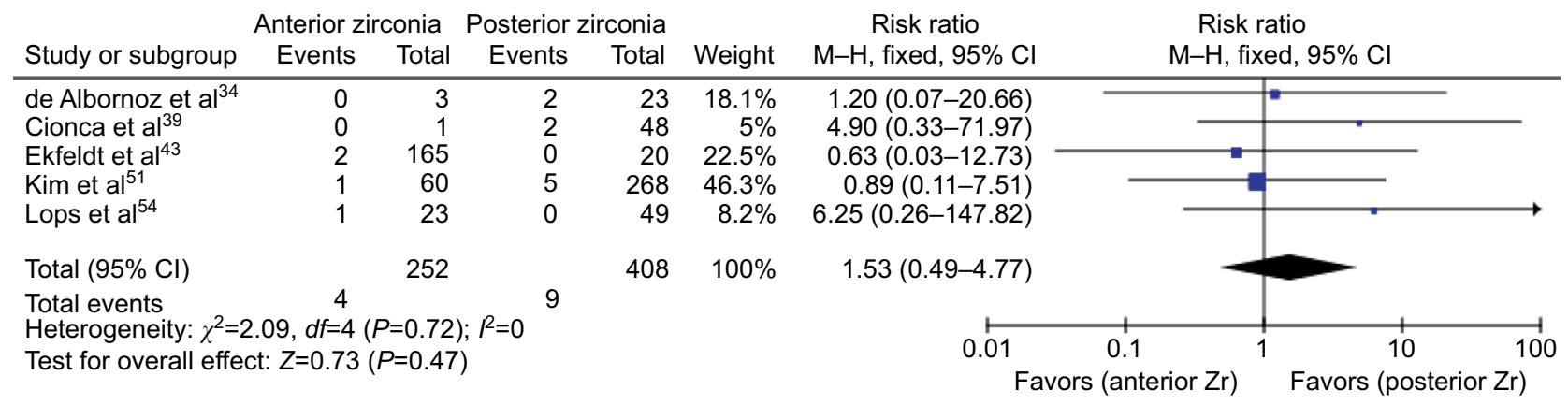

Figure 2 Forest plot of comparison.

Notes: Anterior zirconia versus posterior zirconia. Outcome: abutment failure.

Abbreviation: $\mathrm{M}-\mathrm{H}$, Mantel-Haenszel.

interpretation. Selection and measurement bias will always be present in case series, in addition to potential risk of incorporation bias, to benefit the final outcome of the intervention. Other than the low number of RCTs, one of the major shortcomings of the reviewed literature was the potential language bias in our study, as we considered only literature written in English.

A further limitation of the study was the lack of data regarding the exact material composition of titanium and zirconia abutments used in the included studies, which could have been helpful to verify if there were a correlation between the abutment failures manifested and the actual material composition of the zirconia abutment. However, this information was lacking in the reviewed literature, due to the fact that most manufacturers do not usually disclose such information.

Due to the diversity of parameters, lack of standardized methods, and the heterogeneity of the included studies, the results of our study require cautious interpretation. Highlevel evidence-based comparative studies are needed to demonstrate outcomes of abutment materials in the anterior region compared to the posterior region. The next step for 


\begin{tabular}{|c|c|c|c|c|}
\hline \multicolumn{4}{|l|}{ Posterior } & \multirow{2}{*}{$\begin{array}{l}\text { Other } \\
\text { Notes }\end{array}$} \\
\hline $\begin{array}{l}\text { Technical } \\
\text { complications }\end{array}$ & Prosthetic complications & Biological complications & $\begin{array}{l}\text { Aesthetic } \\
\text { complications }\end{array}$ & \\
\hline- & - & - & - & \\
\hline $\begin{array}{l}\text { I implant lost } 8 \text { months } \\
\text { after restoration }(\mathrm{Zr})\end{array}$ & None & None & None & \\
\hline $\begin{array}{l}3 \text { implants lost in the } \\
\text { same patient after } \\
\text { loading, but before final } \\
\text { restoration ( } \mathrm{Zr}, 2 \mathrm{Ti})\end{array}$ & $\begin{array}{l}3 \text { minor chipping of porcelain } \\
(\mathrm{Zr})\end{array}$ & None & NR & \\
\hline- & - & - & - & \\
\hline None & None & None & NR & \\
\hline None & None & None & NR & \\
\hline $\begin{array}{l}3 \text { implant failures due to } \\
\text { loss of osseointegration } \\
(2 \mathrm{Zr}, \mathrm{ITi})\end{array}$ & $\begin{array}{l}2 \text { minor chipping of porcelain } \\
\text { (Ti) }\end{array}$ & None & None & \\
\hline None & None & None & NR & \\
\hline 10 implants lost $(\mathrm{Zr}) / 4$ & 3 loss of crown retention/29 & 4 fistula (I Zr, $3 \mathrm{Ti})$, suppuration & & \\
\hline implants lost $(\mathrm{Ti}) / 24$ & minor chipping & on probing and pocket depth & & \\
\hline abutment loosening & & $\geq 5 \mathrm{~mm}$ & & \\
\hline$(\mathrm{Zr}) / \mathrm{I}$ screw fracture & & & & \\
\hline$(\mathrm{Zr}) / 2$ abutment & & & & \\
\hline loosening $(\mathrm{Ti})$ & & & & \\
\hline
\end{tabular}

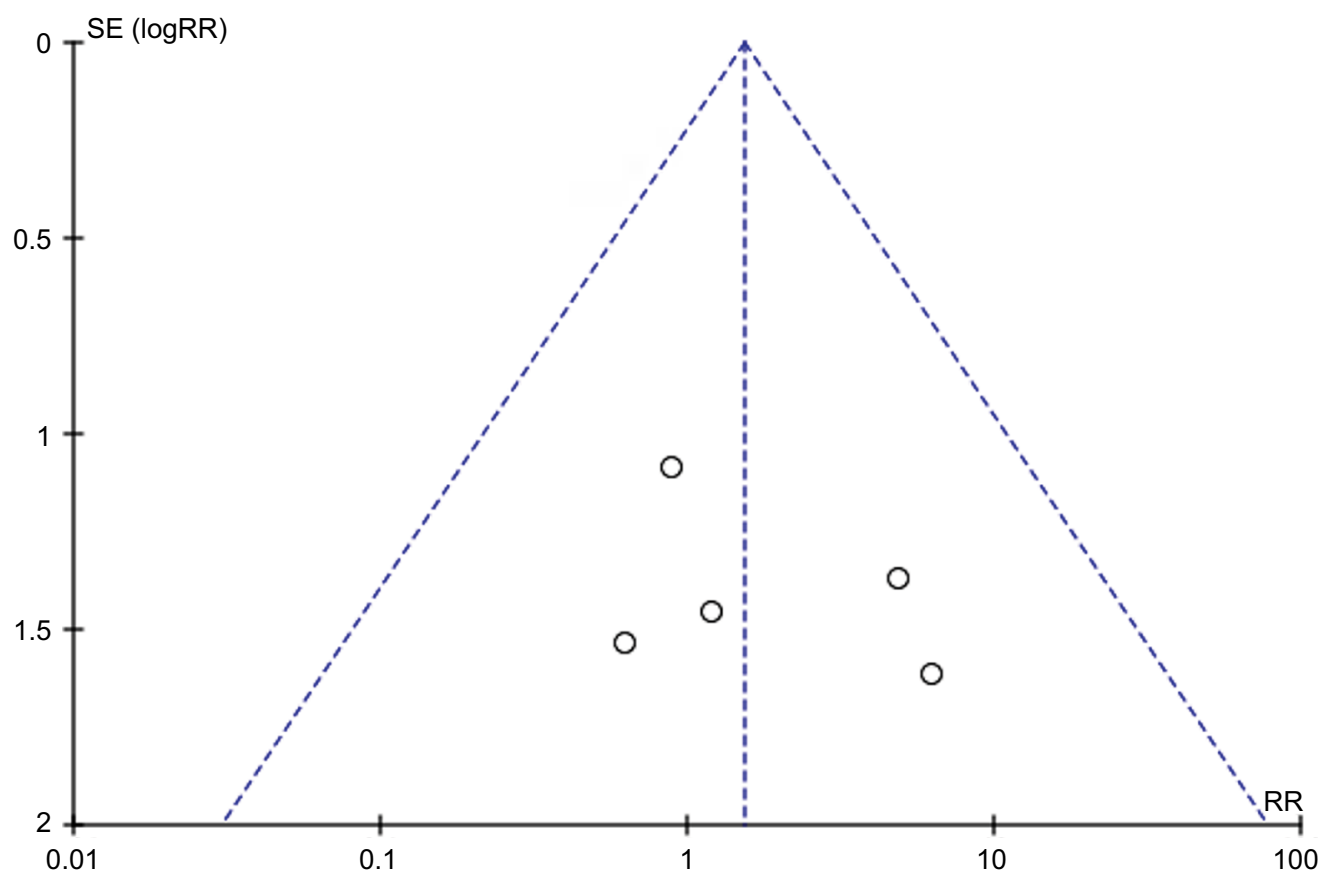

Figure 3 Funnel plot of comparison.

Notes: Anterior versus posterior zirconia. Outcome: abutment failure.

Abbreviations: SE, standard error; RR, risk ratio. 


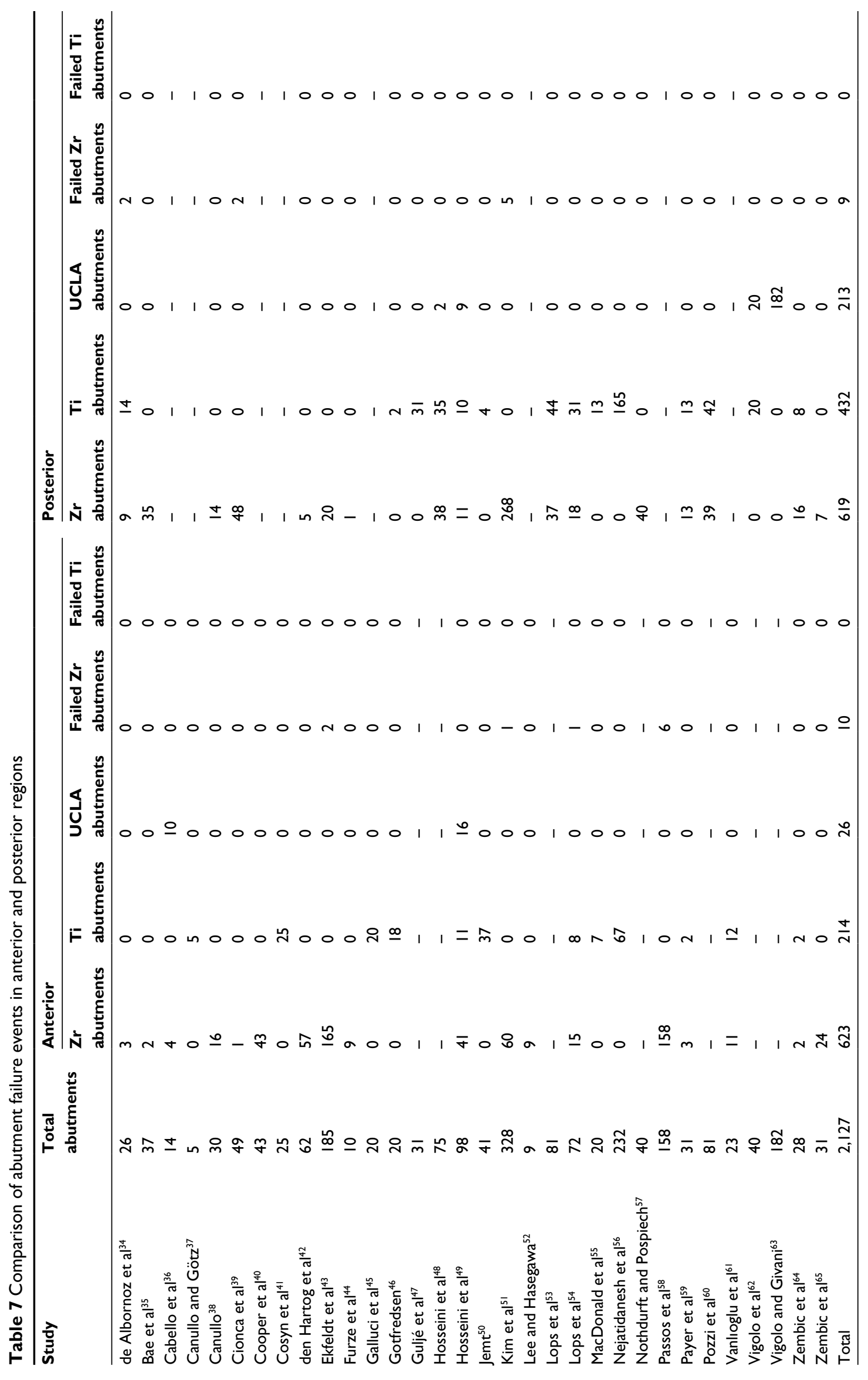


future clinical trials should be to compare directly the performance of an implant-abutment material in the anterior and posterior regions.

\section{Conclusion}

There were no reported failures of titanium or UCLA abutments. For failures of zirconia abutments, a meta-analysis showed no significant differences in zirconia-abutment failure between anterior and posterior regions. However, technical complications were more commonly reported in posterior locations and commonly reported in zirconia abutments. Abutment-screw loosening was the most common technical complication, while prosthetic, biological, and aesthetic complications showed insignificant differences irrespective to the abutment material used.

\section{Acknowledgments}

This work was financially supported by the National Natural Science Foundation of China (81500894) and the Specialized Research Fund for the Doctoral Program of China (SRFDP20125503120009) to SY, Program for Innovation Team Building at Institutions of Higher Education in Chongqing in 2016 and Chongqing Municipal Key Laboratory of Oral Biomedical Engineering of Higher Education.

\section{Disclosure}

The authors report no conflicts of interest in this work.

\section{References}

1. Lekholm U, Gunne J, Henry P, et al. Survival of the Brånemark implant in partially edentulous jaws: a 10-year prospective multicenter study. Int J Oral Maxillofac Implants. 1999;14(5):639-645.

2. Adell R, Lekholm U, Rockler B, Brånemark PI. A 15-year study of osseointegrated implants in the treatment of the edentulous jaw. Int $J$ Oral Surg. 1981;10(6):387-416.

3. Holst S, Blatz MB, Hegenbarth E, Wichmann M, Eitner S. Prosthodontic considerations for predictable single-implant esthetics in the anterior maxilla. J Oral Maxillofac Surg. 2005;63(9 Suppl 2):89-96.

4. Zembic A, Bösch A, Jung RE, Hämmerle CH, Sailer I. Five-year results of a randomized controlled clinical trial comparing zirconia and titanium abutments supporting single-implant crowns in canine and posterior regions. Clin Oral Implants Res. 2013;24(4):384-390.

5. Paphangkorakit J, Osborn JW. The effect of pressure on a maximum incisal bite force in man. Arch Oral Biol. 1997;42(1):11-17.

6. Ferrario VF, Sforza C, Serrao G, Dellavia C, Tartaglia GM. Single tooth bite forces in healthy young adults. J Oral Rehabil. 2004;31(1):18-22.

7. Nishigawa K, Bando E, Nakano M. Quantitative study of bite force during sleep associated bruxism. J Oral Rehabil. 2001;28(5):485-491.

8. Sailer I, Philipp A, Zembic A, Pjetursson BE, Hämmerle CH, Zwahlen M. A systematic review of the performance of ceramic and metal implant abutments supporting fixed implant reconstructions. Clin Oral Implants Res. 2009;20 Suppl 4:4-31.

9. Zembic A, Kim S, Zwahlen M, Kelly JR. Systematic review of the survival rate and incidence of biologic, technical, and esthetic complications of single implant abutments supporting fixed prostheses. Int $J$ Oral Maxillofac Implants. 2014;29 Suppl:99-116.
10. Bidra AS, Rungruanganunt P. Clinical outcomes of implant abutments in the anterior region: a systematic review. $J$ Esthet Restor Dent. 2013;25(3):159-176.

11. Vechiato-Filho AJ, Pesqueira AA, De Souza GM, dos Santos DM, Pellizzer EP, Goiato MC. Are zirconia implant abutments safe and predictable in posterior regions? A systematic review and meta-analysis. Int J Prosthodont. 2016;29(3):233-244.

12. Higgins JP, Green S, editors. Cochrane Handbook for Systematic Reviews of Interventions. London: Cochrane Collaboration; 2011.

13. Wells GA, Shea BJ, O'Connell D, et al. The Newcastle-Ottawa Scale (NOS) for assessing the quality of nonrandomised studies in metaanalyses. 2014. Available from: http://www.ohri.ca/programs/clinical_epidemiology/oxford.asp. Accessed July 1, 2016.

14. den Hartog L, Slater JJ, Vissink A, Meijer HJ, Raghoebar GM. Treatment outcome of immediate, early and conventional single-tooth implants in the aesthetic zone: a systematic review to survival, bone level, soft-tissue, aesthetics and patient satisfaction. J Clin Periodontol. 2008;35(12):1073-1086.

15. Bonde MJ, Stokholm R, Isidor F, Schou S. Outcome of implantsupported single-tooth replacements performed by dental students: a 10-year clinical and radiographic retrospective study. Eur J Oral Implantol. 2010;3(1):37-46.

16. Bressan E, Paniz G, Lops D, Corazza B, Romeo E, Favero G. Influence of abutment material on the gingival color of implant-supported all-ceramic restorations: a prospective multicenter study. Clin Oral Implants Res. 2011;22(6):631-637.

17. Büchi DL, Sailer I, Fehmer V, Hämmerle CH, Thoma DS. All-ceramic single-tooth implant reconstructions using modified zirconia abutments: a prospective randomized controlled clinical trial of the effect of pink veneering ceramic on the esthetic outcomes. Int J Periodontics Restorative Dent. 2014;34(1):29-37.

18. Camargos GV, do Prado CJ, das Neves FD, Sartori IA. Clinical outcomes of single dental implants with external connections: results after 2 to 13 years. Int J Oral Maxillofac Implants. 2012; 27(4):935-944.

19. Cooper LF, Reside G, Stanford C, et al. A multicenter randomized comparative trial of implants with different abutment interfaces to replace anterior maxillary single teeth. Int J Oral Maxillofac Implants. 2015;30(3):622-632.

20. Cosgarea R, Gasparik C, Dudea D, Culic B, Dannewitz B, Sculean A. Peri-implant soft tissue colour around titanium and zirconia abutments: a prospective randomized controlled clinical study. Clin Oral Implants Res. 2015;26(5):537-544.

21. Deporter D, Ogiso B, Sohn DS, Ruljancich K, Pharoah M. Ultrashort sintered porous-surfaced dental implants used to replace posterior teeth. J Periodontol. 2008;79(7):1280-1286.

22. Ferrari M, Cagidiaco MC, Garcia-Godoy F, Goracci C, Cairo F. Effect of different prosthetic abutments on peri-implant soft tissue: a randomized controlled clinical trial. Am J Dent. 2015;28(2):85-89.

23. Happe A, Schulte-Mattler V, Fickl S, Naumann M, Zöller JE, Rothamel D. Spectrophotometric assessment of peri-implant mucosa after restoration with zirconia abutments veneered with fluorescent ceramic: a controlled, retrospective clinical study. Clin Oral Implants Res. 2013;24 Suppl A100:28-33.

24. Jung DW, Yoon HJ. Clinical and retrospective evaluation of 4.1- or 4.3-mm-diameter implants placed immediately in the molar region: a preliminary study. J Oral Maxillofac Surg. 2016;74(3):489-496.

25. Kreissl ME, Gerds T, Muche R, Heydecke G, Strub JR. Technical complications of implant-supported fixed partial dentures in partially edentulous cases after an average observation period of 5 years. Clin Oral Implants Res. 2007;18(6):720-726.

26. Payer M, Arnetzl V, Kirmeier R, Koller M, Arnetzl G, Jakse N. Immediate provisional restoration of single-piece zirconia implants - a prospective case series: results after 24 months of clinical function. Clin Oral Implants Res. 2013;24(5):569-575.

27. Pettersson P, Sennerby L. A 5-year retrospective study on Replace Select Tapered dental implants. Clin Implant Dent Relat Res. 2015;17(2): 286-295. 
28. Redemagni M, Cremonesi S, Garlini G, Maiorana C. Soft tissue stability with immediate implants and concave abutments. Eur J Esthet Dent. 2009;4(4):328-337.

29. Rompen E, Raepsaet N, Domken O, Touati B, Van Dooren E. Soft tissue stability at the facial aspect of gingivally converging abutments in the esthetic zone: a pilot clinical study. J Prosthet Dent. 2007;97(6 Suppl):S119-S125.

30. van Brakel R, Noordmans HJ, Frenken J, de Roode R, de Wit GC, Cune MS. The effect of zirconia and titanium implant abutments on light reflection of the supporting soft tissues. Clin Oral Implants Res. 2011;22(10): $1172-1178$

31. van Brakel R, Meijer GJ, Verhoeven JW, Jansen J, de Putter C, Cune MS. Soft tissue response to zirconia and titanium implant abutments: an in vivo within-subject comparison. $J$ Clin Periodontol. 2012;39(10):995-1001.

32. Vanlıŏlu BA, Kahramanoğlu E, Yıldız C, Ozkan Y, Kulak-Özkan Y. Esthetic outcome evaluation of maxillary anterior single-tooth bonelevel implants with metal or ceramic abutments and ceramic crowns. Int J Oral Maxillofac Implants. 2014;29(5):1130-1136.

33. Visser A, Raghoebar GM, Meijer HJ, Meijndert L, Vissink A. Care and aftercare related to implant-retained dental crowns in the maxillary aesthetic region: a 5-year prospective randomized clinical trial. Clin Implant Dent Relat Res. 2011;13(2):157-167.

34. de Albornoz AC, Vignoletti F, Ferrantino L, Cárdenas E, De Sanctis M, Sanz M. A randomized trial on the aesthetic outcomes of implantsupported restorations with zirconia or titanium abutments. $J$ Clin Periodontol. 2014;41(12):1161-1169.

35. Bae KH, Han JS, Seol YJ, Butz F, Caton J, Rhyu IC. The biologic stability of alumina-zirconia implant abutments after 1 year of clinical service: a digital subtraction radiographic evaluation. Int J Periodontics Restorative Dent. 2008;28(2):137-143.

36. Cabello G, Rioboo M, Fábrega JG. Immediate placement and restoration of implants in the aesthetic zone with a trimodal approach: soft tissue alterations and its relation to gingival biotype. Clin Oral Implants Res. 2013;24(10):1094-1100.

37. Canullo L, Götz W. Peri-implant hard tissue response to glow-discharged abutments - prospective study: preliminary radiological results. Ann Anat. 2012;194(6):529-532.

38. Canullo L. Clinical outcome study of customized zirconia abutments for single-implant restorations. Int J Prosthodont. 2007;20(5):489-493.

39. Cionca N, Müller N, Mombelli A. Two-piece zirconia implants supporting all-ceramic crowns: a prospective clinical study. Clin Oral Implants Res. 2015;26(4):413-418.

40. Cooper LF, Ellner S, Moriarty J, et al. Three-year evaluation of singletooth implants restored 3 weeks after 1-stage surgery. Int J Oral Maxillofac Implants. 2007;22(5):791-800.

41. Cosyn J, Eghbali A, De Bruyn H, Collys K, Cleymaet R, De Rouck T. Immediate single-tooth implants in the anterior maxilla: 3-year results of a case series on hard and soft tissue response and aesthetics. J Clin Periodontol. 2011;38(8):746-753.

42. den Hartog L, Raghoebar GM, Stellingsma K, Vissink A, Meijer HJ. Immediate non-occlusal loading of single implants in the aesthetic zone: a randomized clinical trial. J Clin Periodontol. 2011;38(2):186-194.

43. Ekfeldt A, Fürst B, Carlsson GE. Zirconia abutments for single-tooth implant restorations: a retrospective and clinical follow-up study. Clin Oral Implants Res. 2011;22(11):1308-1314.

44. Furze D, Byrne A, Donos N, Mardas N. Clinical and esthetic outcomes of single-tooth implants in the anterior maxilla. Quintessence Int. 2012;43(2):127-134.

45. Gallucci GO, Grütter L, Nedir R, Bischof M, Belser UC. Esthetic outcomes with porcelain-fused-to-ceramic and all-ceramic singleimplant crowns: a randomized clinical trial. Clin Oral Implants Res. 2011;22(1):62-69.

46. Gotfredsen K. A 10-year prospective study of single tooth implants placed in the anterior maxilla. Clin Implant Dent Relat Res. 2012;14(1): $80-87$.
47. Guljé FL, Raghoebar GM, Vissink A, Meijer HJ. Single restorations in the resorbed posterior mandible supported by 6-mm implants: a 1-year prospective case series study. Clin Implant Dent Relat Res. 2015;17 Suppl 2:e465-e471.

48. Hosseini M, Worsaae N, Schiødt M, Gotfredsen K. A 1-year randomised controlled trial comparing zirconia versus metal-ceramic implant supported single-tooth restorations. Eur J Oral Implantol. 2011;4(4):347-361.

49. Hosseini M, Worsaae N, Schiødt M, Gotfredsen K. A 3-year prospective study of implant-supported, single-tooth restorations of all-ceramic and metal-ceramic materials in patients with tooth agenesis. Clin Oral Implants Res. 2013;24(10):1078-1087.

50. Jemt T. Cemented CeraOne and porcelain fused to TiAdapt abutment single-implant crown restorations: a 10-year comparative follow-up study. Clin Implant Dent Relat Res. 2009;11(4):303-310.

51. Kim SS, Yeo IS, Lee SJ, et al. Clinical use of alumina-toughened zirconia abutments for implant-supported restoration: prospective cohort study of survival analysis. Clin Oral Implants Res. 2013;24(5):517-522.

52. Lee CY, Hasegawa H. Immediate load and esthetic zone considerations to replace maxillary incisor teeth using a new zirconia implant abutment in the bone grafted anterior maxilla. J Oral Implantol. 2008;34(5):259-267.

53. Lops D, Bressan E, Chiapasco M, Rossi A, Romeo E. Zirconia and titanium implant abutments for single-tooth implant prostheses after 5 years of function in posterior regions. Int J Oral Maxillofac Implants. 2013;28(1):281-287.

54. Lops D, Bressan E, Parpaiola A, Sbricoli L, Cecchinato D, Romeo E. Soft tissues stability of cad-cam and stock abutments in anterior regions: 2-year prospective multicentric cohort study. Clin Oral Implants Res. 2015;26(12):1436-1442.

55. MacDonald K, Pharoah M, Todescan R, Deporter D. Use of sintered porous-surfaced dental implants to restore single teeth in the maxilla: a 7- to 9-year follow-up. Int $J$ Periodontics Restorative Dent. 2009;29(2):191-199.

56. Nejatidanesh F, Moradpoor H, Savabi O. Clinical outcomes of zirconiabased implant- and tooth-supported single crowns. Clin Oral Investig. 2016;20(1):169-178.

57. Nothdurft F, Pospiech P. Prefabricated zirconium dioxide implant abutments for single-tooth replacement in the posterior region: evaluation of peri-implant tissues and superstructures after 12 months of function. Clin Oral Implants Res. 2010;21(8):857-865.

58. Passos SP, Linke B, Larjava H, French D. Performance of zirconia abutments for implant-supported single-tooth crowns in esthetic areas: a retrospective study up to 12-year follow-up. Clin Oral Implants Res. 2016;27(1):47-54.

59. Payer M, Heschl A, Koller M, Arnetzl G, Lorenzoni M, Jakse N. All-ceramic restoration of zirconia two-piece implants: a randomized controlled clinical trial. Clin Oral Implants Res. 2015;26(4):371-376.

60. Pozzi A, Sannino G, Barlattani A. Minimally invasive treatment of the atrophic posterior maxilla: a proof-of-concept prospective study with a follow-up of between 36 and 54 months. $J$ Prosthet Dent. 2012;108(5):286-297.

61. Vanloglu BA, Özkan Y, Evren B, Özkan YK. Experimental custommade zirconia abutments for narrow implants in esthetically demanding regions: a 5-year follow-up. Int J Oral Maxillofac Implants. 2012;27(5):1239-1242.

62. Vigolo P, Givani A, Majzoub Z, Cordioli G. A 4-year prospective study to assess peri-implant hard and soft tissues adjacent to titanium versus gold-alloy abutments in cemented single implant crowns. J Prosthodont. 2006;15(4):250-256.

63. Vigolo P, Givani A. Platform-switched restorations on wide-diameter implants: a 5-year clinical prospective study. Int J Oral Maxillofac Implants. 2009;24(1):103-109.

64. Zembic A, Bösch A, Jung RE, Hämmerle CH, Sailer I. Five-year results of a randomized controlled clinical trial comparing zirconia and titanium abutments supporting single-implant crowns in canine and posterior regions. Clin Oral Implants Res. 2013;24(4):384-390. 
65. Zembic A, Philipp AO, Hämmerle CH, Wohlwend A, Sailer I. Elevenyear follow-up of a prospective study of zirconia implant abutments supporting single all-ceramic crowns in anterior and premolar regions. Clin Implant Dent Relat Res. 2015;17 Suppl 2:e417-e426.

66. Quek CE, Tan KB, Nicholls JI. Load fatigue performance of a singletooth implant abutment system: effect of diameter. Int J Oral Maxillofac Implants. 2006;21(6):929-936.

67. Quek HC, Tan KB, Nicholls JI. Load fatigue performance of four implant-abutment interface designs: effect of torque level and implant system. Int J Oral Maxillofac Implants. 2008;23(2):253-262.

68. Aramouni P, Zebouni E, Tashkandi E, Dib S, Salameh Z, Almas K. Fracture resistance and failure location of zirconium and metallic implant abutments. J Contemp Dent Pract. 2008;9(7):41-48.

69. Seetoh YL, Tan KB, Chua EK, Quek HC, Nicholls JI. Load fatigue performance of conical implant-abutment connections. Int $\mathrm{J}$ Oral Maxillofac Implants. 2011;26(4):797-806.
70. Adatia ND, Bayne SC, Cooper LF, Thompson JY. Fracture resistance of yttria-stabilized zirconia dental implant abutments. J Prosthodont. 2009;18(1):17-22.

71. Graziani F, Donos N, Needleman I, Gabriele M, Tonetti M. Comparison of implant survival following sinus floor augmentation procedures with implants placed in pristine posterior maxillary bone: a systematic review. Clin Oral Implants Res. 2004;15(6):677-682.

72. Tolstunov L. Implant zones of the jaws: implant location and related success rate. J Oral Implantol. 2007;33(4):211-220.

73. Kohal RJ, Weng D, Bächle M, Strub JR. Loaded custom-made zirconia and titanium implants show similar osseointegration: an animal experiment. J Periodontol. 2004;75(9):1262-1268.

74. Linkevicius T, Vaitelis J. The effect of zirconia or titanium as abutment material on soft peri-implant tissues: a systematic review and metaanalysis. Clin Oral Implants Res. 2015;26 Suppl 11:139-147.
Clinical, Cosmetic and Investigational Dentistry

\section{Publish your work in this journal}

Clinical, Cosmetic and Investigational Dentistry is an international peer-reviewed, open access, online journal focusing on the latest clinical and experimental research in dentistry with specific emphasis on cosmetic interventions. Innovative developments in dental materials, techniques and devices that improve outcomes and patient satisfac-

\section{Dovepress}

tion and preference will be highlighted. The manuscript management system is completely online and includes a very quick and fair peerreview system, which is all easy to use. Visit http://www.dovepress. com/testimonials.php to read real quotes from published authors. 